NUCLEAR MATERIALS

Author(s):

M. M. Pickrell

Submitted to:

Mid-Year Progress Report

(PROGRESS REPORT)

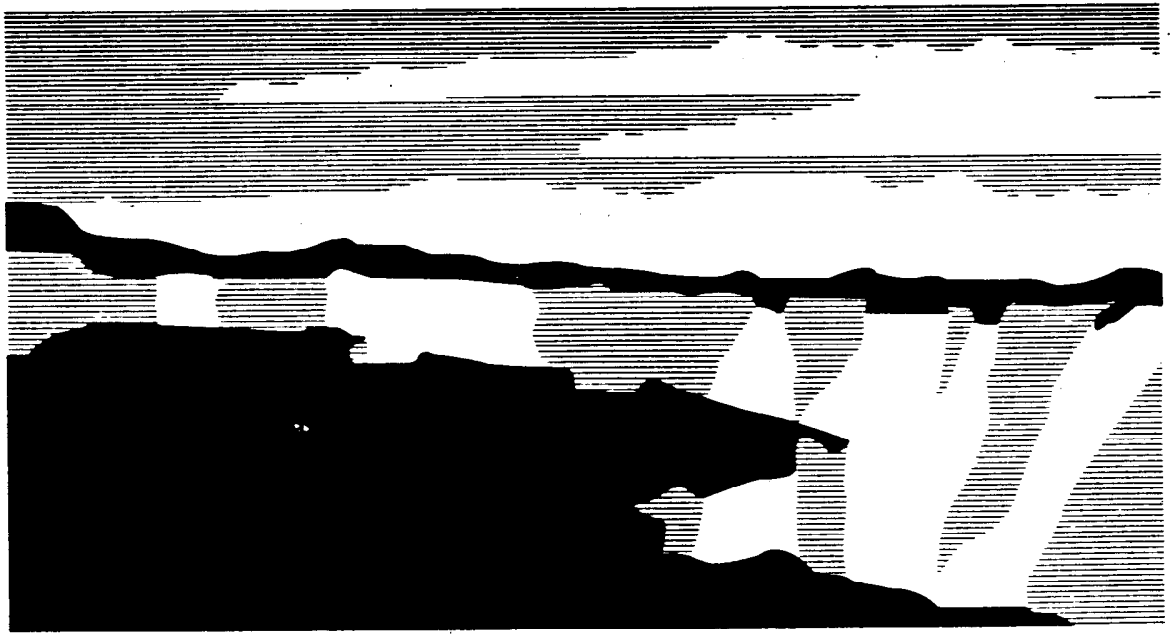

Los Alamos National Laboratory, an affirmative actionequal opportunity employer, is operated by the University of Calitornia for the U.S. Department of Energy under contract W-7405-ENG-36. By accoptance of this articte, the publisher recognizes that the U.S. Government retains a nonexclusive, royalty-free license to publish or reproduce the pubtished form of this contribution, or to allow others to do so, for U.S. Governmem purposes. The Los Alamos National Laboratory requests that the publisher identity this article as work pertormed under the auspices of the U.S. Department of Energy. 


\title{
High Fluence Neutron Source for Nondestructive Characterization of Nuclear Materials
}

\author{
Mid-Year Progress Report
}

\author{
Mark M. Pickrell \\ Los Alamos National Laboratory
}

J une 1, 1997

\section{Goals of Project:}

We are addressing the need to measure nuclear wastes, residues, and spent fuel in order to process these for final disposition. For example, TRU wastes destined for the WIPP must satisfy extensive characterization criteria outlined in the Waste Acceptance Criteria, the Quality Assurance Program Plan, and the Performance Demonstration Plan. Similar requirements exist for spent fuel and residues. At present, no nondestructive assay instrumentation is capable of satisfying all of the PDP test cycles. One of the primary methods for waste assay is by active neutron intezrooation.

We plan to improve the capability of all active neutron systems by providing a higher intensity neutron source (by about a factor of 1,000) for essentially the same cost, power, and space requirements as existing systems.

This high intensity neutron source will be an electrostatically confined (IEC) plasma device. The IEC is a symmetric sphere that was originally developed in the $1950 \mathrm{~s}$ as a possible fusion reactor. It operates as D-T neutron generator. Although it was not believed to scale to fusion reactor levels, these experiments demonstrated a neutron yield of $2 \times 10^{10}$ neutrons/second on table-top experiments that could be powered from ordinary laboratory circuits (1 kilowatt). Subsequently, the IEC physics has been extensively studied at the University of Illinois. The basis for scaling the output up to $1 \times 10^{11} \mathrm{n} / \mathrm{s}$ has been established. In addition, IEC devices have run for cumulative times approaching 10,000 hours. They have been operated in pulsed-and continuous mode. The essential features of the IEC plasma neutron source, compared to existing sources of the same cost, size and power consumption, are:

Table I: Present and Target Operating Parameters for Small Neutron Generators

\begin{tabular}{lll}
\hline Parameter & Present & IE C Target or Already Proven \\
\hline Neutron Yield $(\mathrm{n} / \mathrm{s})$ & $10^{8}$ & $10^{11}$ \\
Lifetime (hours) & $\mathbf{5 0 0}$ & 10,000 \\
Operation & Pulsed & Pulsed or steady state \\
Nominal cost \$k & $\$ 100 \mathrm{k}$ & Same \\
Power & $1 \mathrm{~kW}$ & $10 \mathrm{~kW}$ \\
\hline
\end{tabular}




\section{Technical Description of Work:}

The design of a conventional IEC source is deceptively simple. The basic system is a spherical vacuum chamber containing a spherical grid. The grid is raised to a high negative potential. A breakdown develops between the chamber wall and the grid, and this plasma becomes a source of positive deuterium and tritium ions. These ions are accelerated to the center of the vacuum chamber sphere where they may collide. The ion energy may achieve the full potential of the accelerating grid. If the grid is raised to a nominal $100 \mathrm{kv}$, the coulomb barrier for D-T fusion, then the fusion cross section becomes quite large and the neutron production proceeds.

The IEC concept was originally developed in the late 1950's and early 1960's by R. L. Hirsch and collaborators. Its incipient purpose was a possible plasma fusion experiment source for the fusion energy program. The idea was initially presented to the DOE with a table-top experiment using ordinary office power. That system produced in excess of $10^{8}$ neutrons per second. Although the IEC was not favored for a future electric energy generator, the application as a potential neutron source was clearly established. Using nominal laboratory power and a modest sized sphere, Hirsch was able to achieve a maximum neutron yield of $2 \times 10^{10}$ neutrons per second in the mid 1960's.

The achievement of a total neutron yield of $2 \times 10^{10} \mathrm{n} / \mathrm{s}$ was a remarkable result, but the more important contribution of Hirsch was establishing the underlying plasma physics basis of IEC operations. Hirsch found that the IEC neutron yield scaled strongly with grid current, but not strongly with voltage. This result is surprising because ideally the accelerating ions collide with the grid potential energy, and the scaling of the fusion cross section with collision energy is very strong. Hirsch also found that neutron yield scaled inversely with density, to the extent that density could be varied in a static device. This result was very surprising, because the classical reaction rate is proportional to the density squared. Hirsch correctly surmised that the reason for this behavior was the plasma collisionality. The IEC plasma was operating in the collisional regime. In collisional operations, accelerated ions are likely to collide with fill gas neutrals in the accelerating grid interior. Thus the center of mass collision energy is derived from a single particle only, as the neutral is effectively stationary. In addition, each ion probably suffers multiple collisions with the neutral gas, because the Rutherford cross section is largest at low energies. Therefore, the ions never achieve the full accelerating potential of the grid. The fusion cross section drops rapidly with reduced collision energy and the neutron yield is small. In this mode the ions do not collide in a single, tiny point in the center of the chamber, but over a larger contained volume. The multiple collisions can also-import angular momentum to the particles that further increases the collision volume (although the plasma fluid must remain at zero angular momentum).

As density is decreased, (or as current is increased), the plasma collisionality drops. Ions can accelerate more between collisions and achieve higher collision energy. The fusion cross section increases. In addition, the rate of beam-background (or beam-neutral) collisions drops. Less angular momentum is imported and the ions are more tightly focused at the center of the IEC chamber. The beam-beam collision rate increases which effectively doubles the center of mass collision energy. The neutron yield increases with the larger fusion cross section. As the density is dropped, the plasma becomes collisionless, the ions focus in a tight spot at the chamber center, the dominant interaction is beam-beam, and the ions approach the full accelerating potential of the grid. In this mode, neutron production is highest.

These results were demonstrated experimentally by Hirsch 3 decades ago. They have subsequently been confirmed by a 1-1/2 dimensional, fully kinetic, plasma simulation code with complete atomic physics included. The code results, completed at Los Alamos, have confirmed the underlying dynamics of Hirsch's model. 
However, there are practical and physics reasons why reduced-density IEC plasma experiments have not been deployed.. Fundamental is that the plasma density is not a free variable but is constrained by the Paschen breakdown curve. The Paschen curve relates the applied electric field to the plasma density at breakdown, and constrains the density because without breakdown there is no plasma source of ions. Conventional, single grid, IEC experiments have been limited to high density operation by the Paschen limit. Typically fill gas pressures have been several millitorr.

A novel approach proposed at Los Alamos removes this limitation. The Los Alamos IEC uses a triple grid design. In the triple grid IEC device, the inner grid is the accelerating grid. It is raised to high negative potential and serves the same function as the single grid in conventional IEC systems. The central grid serves as electrical isolation, and is held at ground potential.. The outer grid is raised to a modest positive potential, say 200 volts. Dispenser cathodes around the vacuum chamber wall inject electrons. The electrons are trapped and orbit around the outer grid, ionizing a local plasma. Because of the modest potential, the breakdown occurs at a different point on the Paschen curve, at a much lower density. The limit is further relaxed by the injected ionization from the dispenser cathodes. The result is a lower density plasma. Typical fill pressures for the triple grid IEC devices well over an order of magnitude below their single grid counterparts. The low density plasma diffuses across the second grid, and is rapidly accelerated by the inner grid. The result is a tight focus of fully accelerated ions that collide in a beam-beam mode. The collision energy and neutron yield are large.

The triple-grid design enables extending Hirsch's results by mitigating the Paschen breakdown limit and operating in the collisionless, beam-beam mode. A schematic of the triple-grid device is shown in figure 1. 
2 (Emitter \#2,

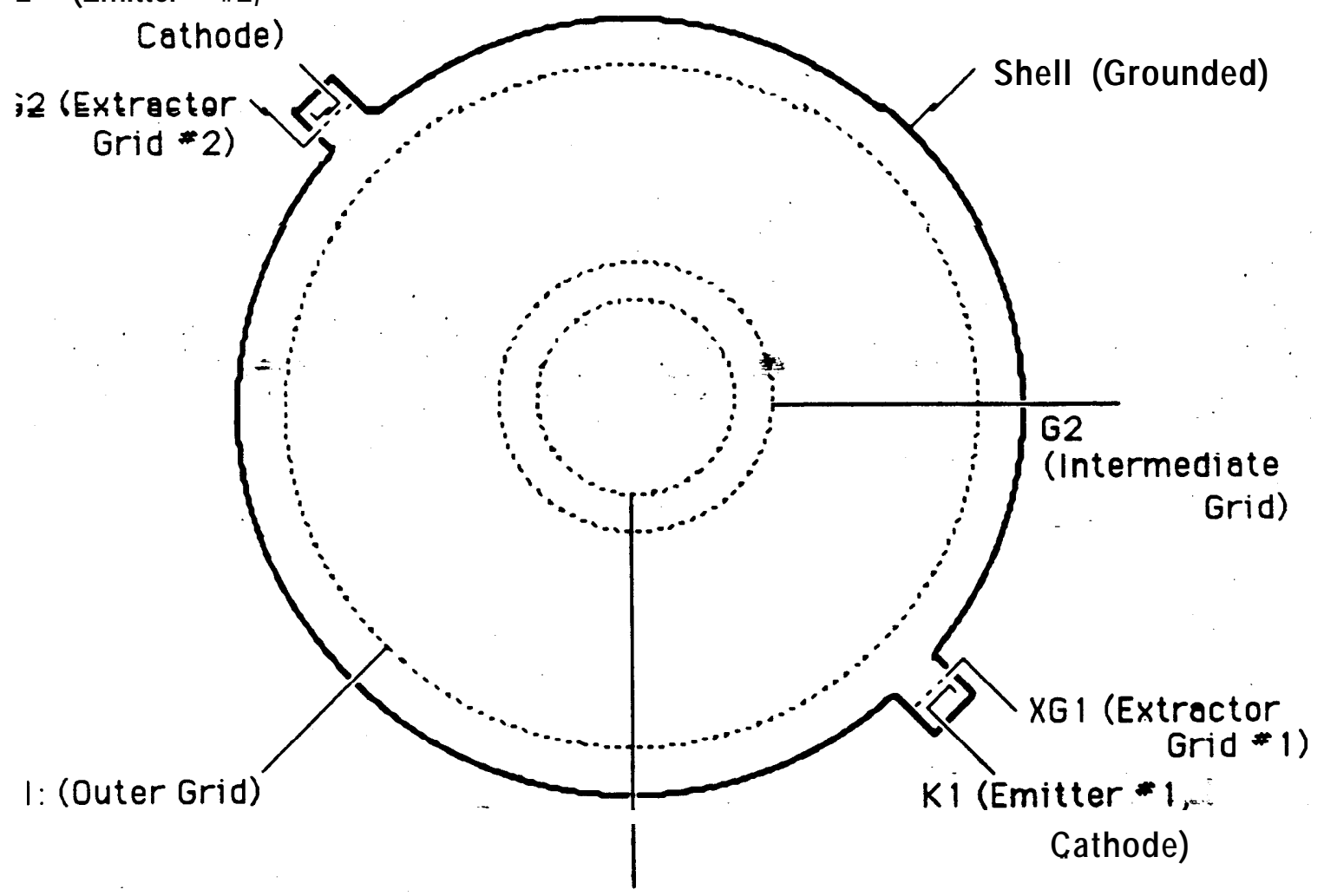

G3 (Central Cathode)

Figure 1: A triple grid IEC schematic.

This approach has been examined at Los Alamos using the fully kinetic simulation code. The code confirms low density breakdown, a collisionless plasma, a tight beam focus, negligible particle angular momentum, and high energy, beam-beam collisions. Low power experiments with triple grid systems have also confirmed the basic scaling relationships. These efforts involved very modest funding level from Los Alamos internal funds. A full, high-power experiment is the next step and is the stage for which we are requesting funding.

The approach for this research project is to construct and test a high-power, triple-grid, IEC experiment according to the specification of the kinetic code simulation.

We believe that a triple grid system can extend Hirsch's 1960's results a factor of 5 to achieve the $10^{11} \mathrm{n} / \mathrm{s}$ target for this proposal. Table 2 summarizes presently achieved IEC performance and our target values. The extrapolations are reasonable given the significant advances in plasma physics, power electronics, and instrumentation in the last 3 decades. 
Table 2: Target and Present IEC Parameters

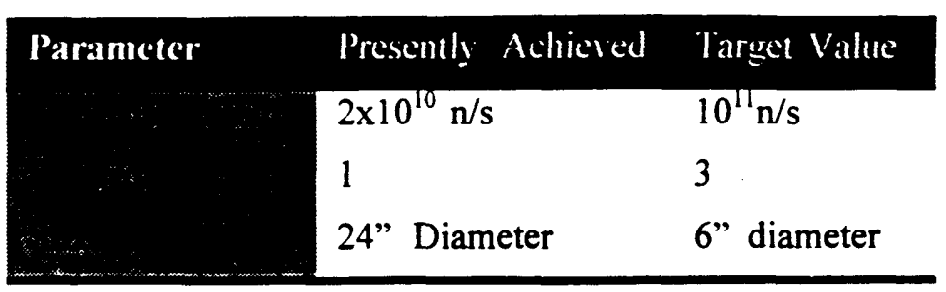

In addition, our objective is. to achieve the modest cost, size, weight and power consumption listed in table I, and the long operating lifetime also listed in table 1 . These values have already been achieved in low power IEC devices at INEL and the University of Illinois. The -scientific challenge will be to maintain these parameters in a high-yield IEC device (except for power which will increase from $1 \mathrm{~kW}$ to $10 \mathrm{~kW}$.). We believe that the low density, triple grid design affords this opportunity.

The scientific justification for this extrapolation is predicated on the basic plasma physics established by Hirsch, and subsequently verified. The collisionality scaling argument is compelling that the target values can be achieved. In addition, the plasma physics computational capability available today for exceeds that 3 decades ago. The fully kinetic code is a highly accurate simulation that will be used to optimize the IEC design for maximum yield and lowest possible power consumption. These analytical tools have only been recently developed.

These are 3 basic tasks outlined for this project, divided into 3 project years. In the first year we will construct the I\&C device. The principal components will be the spherical vacuum chamber containing 3 grids and dispenser cathodes, the main high voltage accelerating power supply (rated at $100 \mathrm{Jv}$ and 100 milliamperes), the intermediate voltage breakdown supply, and control electronics. We project that the construction of all power, control, vacuum, and tritium handling equipment will take the first year. The system will be designed to handle tritium in a sage manner, and it will operate in a deuterium - tritium gas mix. The, first year will culminate with initial operation of the IEC.

The second task and second project year will begin with extensive operations of the IEC experiment. There will be two experimental objectives: to benchmark the kinetic code design against simulation and to map out the plasma scaling relationships. Using this physics basis, a modified IEC chamber will be designed and built during the second half year. The new chamber will be designed to optimized yield at minimum power.

The third task will entail extensive experimental testing of the optimized IEC machine. We will also map out the operating envelope: The physics and operation of the triple grid IEC will be explored. During the second half of this year we will execute the technology transfer of the IEC system. Experimental designs will be converted to a robust, industrial system, and the IEC will be transferred to an industrial partner. A part of the technology transfer CRADA process, the industrial partner will convert the IEC system from a laboratory experiment to an industrial machine. The IEC will then be available for broad application to EM characterization problems.

\section{Accomplishments to Date:}

There were four major efforts that had to be completed in the first year, all leading to the single goal of completing the construction of the IEC device to the point of initial operation. These efforts were: 
1. Design, construction, and assembly of the mechanical systems. These are primarily the vacuum chamber, vacuum pump system, gas flow handling system, and the support structure.

2. Design and construction of the electrical systems. The electrical systems consist of the power supplies that power the IEC grids and also the power supply control systems. The main high voltage is run steady state, but the ionizing grid and the electron injectors operate in a pulsed mode. The control electronics also contains the pulse mode and current feedback controllers.

3. Preparation of the shield-cell experimental area. Because we. anticipate the production of large number of neutrons, an appropriately shielded experimental area must be used. An uncontaminated "hot cell" was available, but it had to be prepared for the IEC experiment, including the installation of $100 \mathrm{~kW}, 3$ phase, 280 volt electrical power.

4. Negotiation and execution of a cooperative research and development agreement (CRADA) to mitigate the research costs and to expedite the commercialization of the technology to industry.

The design and fabrication of all the mechanical and vacuum systems is complete. We have begun assembly of the components in the hot cell. All of the purchased items have been received. All of the custom components have been machined. The most complicated component was the IEC vacuum chamber. Figure 2 is a picture of the two vacuum chamber halves. The chamber is split into 2 halves in order to insert the accelerating grid structures. The halves are joined with a conventional, copper gasket, conflat flange. Other ports on the vacuum chamber are provided for the accelerating grid feed-throughs, electron injectors, and diagnostics.

We anticipate some re-design and development of the accelerating grid feed-throughs because these must be designed to withstand the high accelerating voltages without arcing inside the vacuum chamber. The present feed-throughs are designed with ceramic insulators.

Most of the remaining components were available as commercial, off-the-shelf components and have been procured. 


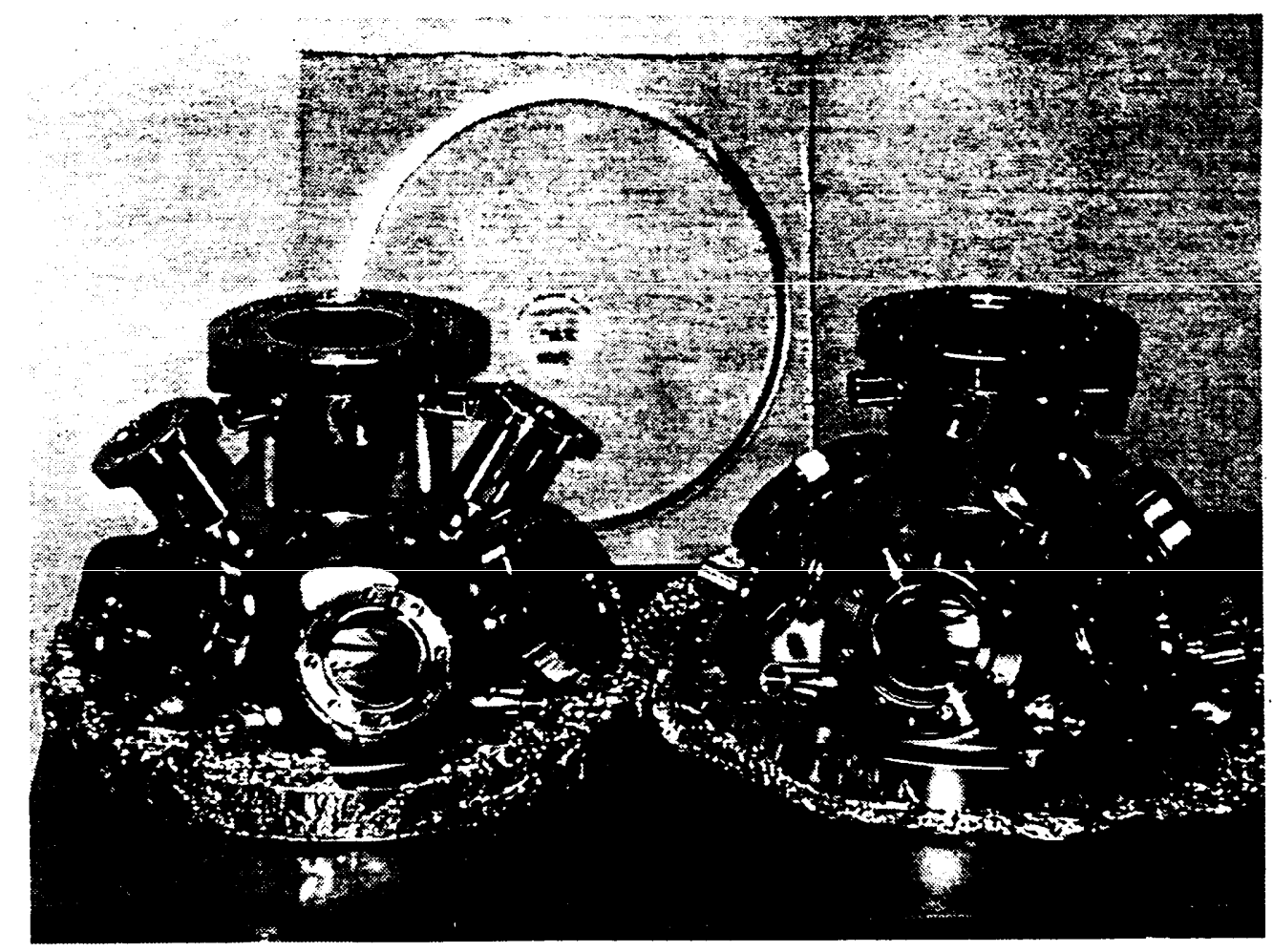

Figure 2: Picture of the two halves of the IEC vacuum chamber.

The design, fabrication, and assembly of the electrical systems is complete. The electrical systems consist of the steady state high voltage power supply for the central grid and the lower voltage, pulsed supply for the ionizing grid. The ionizing grid voltage is regulated and switchable, so that the IEC can be switched on and off rapidly and so that the grid current is maintained. The IEC operates essentially as a vacuum tube, so that the ionizing grid must be energized using a current source. There are also feedback-controlled power supplies for each of the electron injectors. The drive current for each of the injectors is individually regulated and controlled. Again, each injector is operated as a current source. Most of the scaling studies with the IEC will scale either the ionizing grid or electron injector currents.

The electronics system consists of two electronics racks and a high voltage unit. The high voltage unit and one of the racks are the high voltage, 100 kilovolt, 25 kilowatt, accelerating supply for the interior, accelerating grid. The remaining electronics rack contains the power supplies and feedback controllers for the ionizing grid and electron injectors. Figure 3 and 4 shows the complete electronics system and a closeup of the electron injector control panel. 

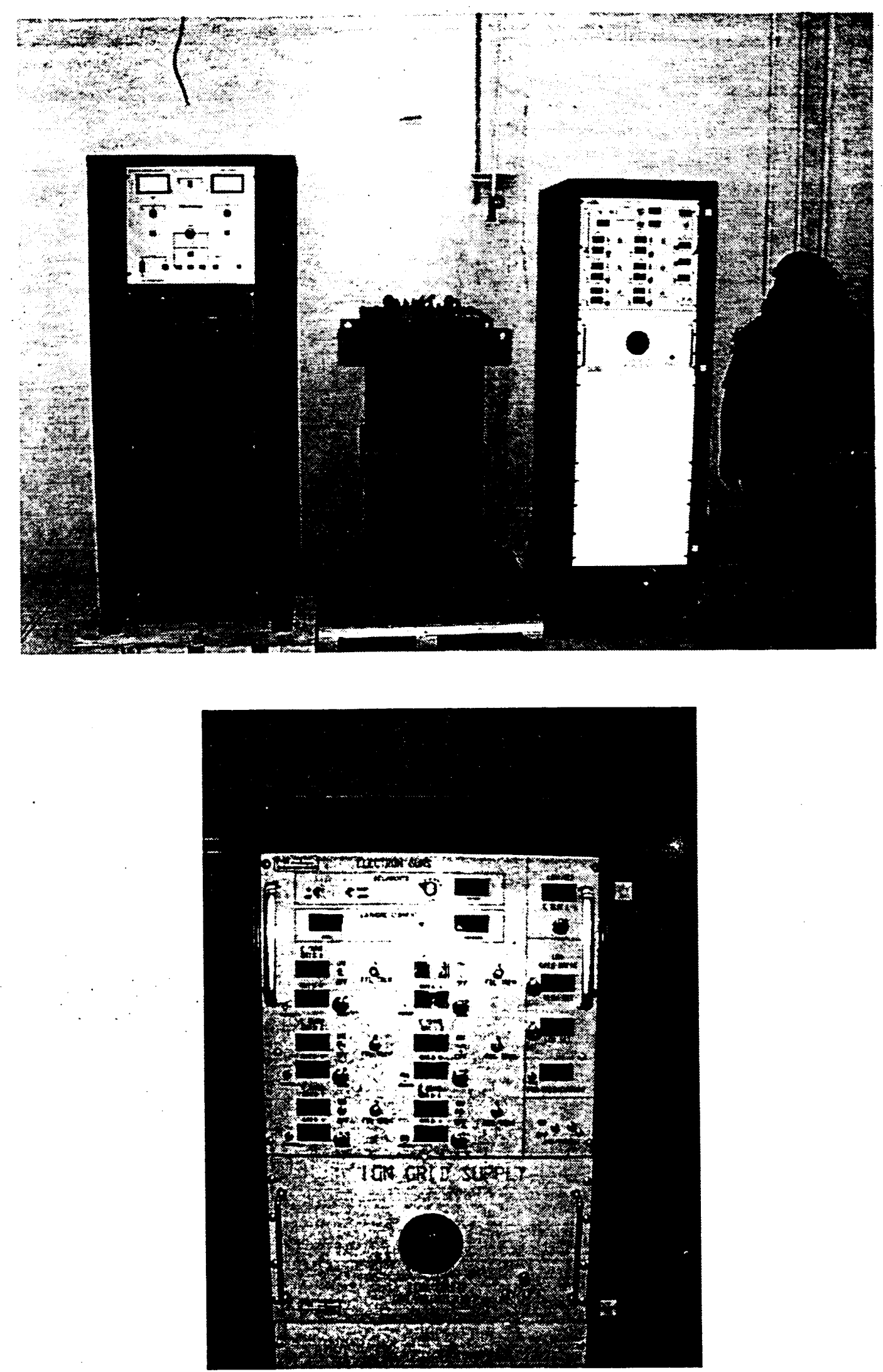
Figures 3 and 4: The upper picture shows the entire electronics system with the technician who assembled the equipment. The lower picture is a close up of the electron injector control panel (right most electronics rack in figure 3).

Figure 5 is a picture of the circuit boards that are used in the feedback controller system and each of the corresponding individual schematics. This system was required in order to operate the ionizing grid and the electron injectors as feedback-controlled, current sources. These cards are plugged into the card cage in the back of the injector control panel.

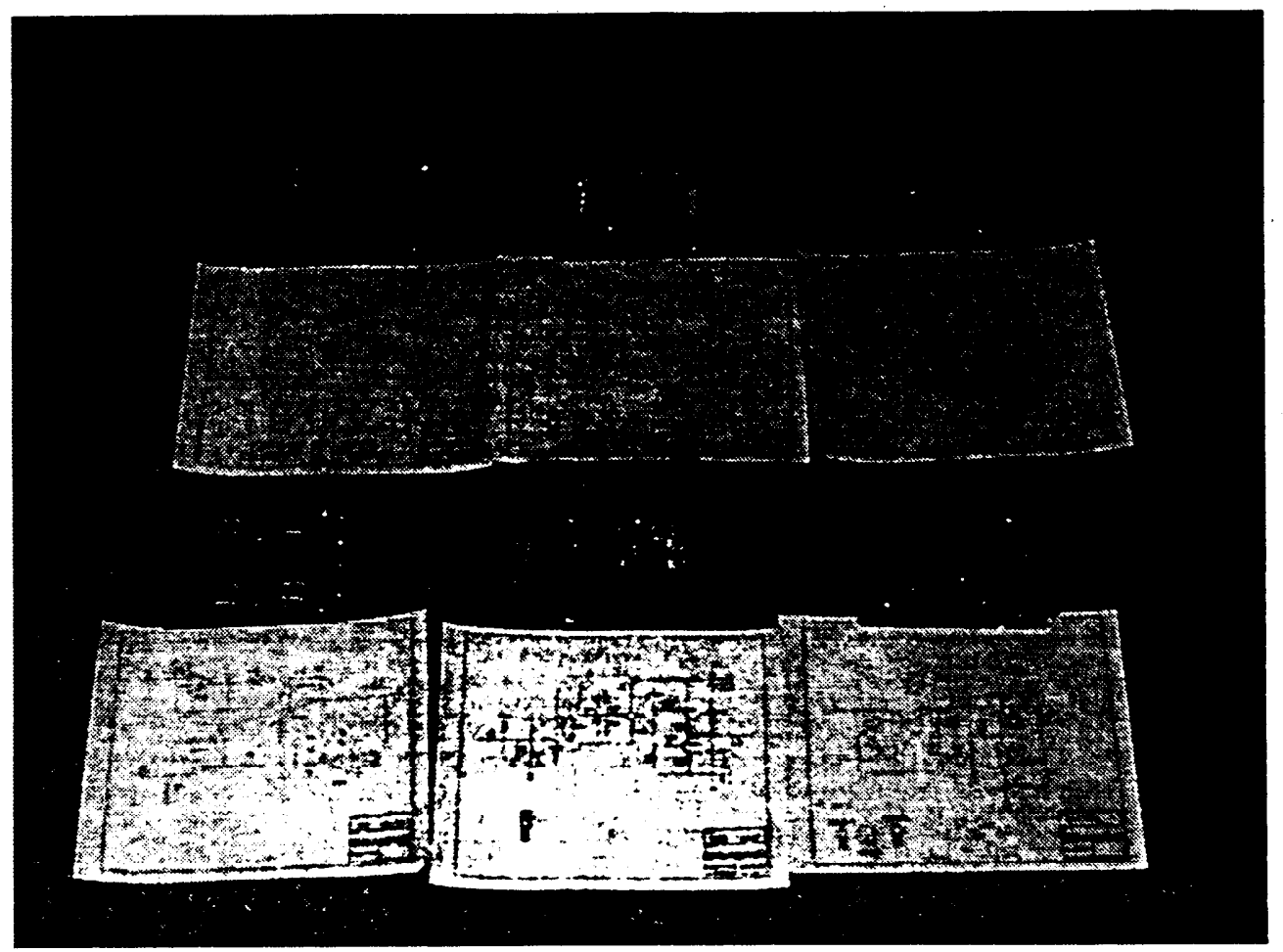

Figure 5: Picture of the circuit boar\& for the feedback controller of the electron injectors and the ionization grid.

The preparation of the "hot-cell" experimental area is complete. The hot cell is necessary to provide adequate shielding from the intense neutron flux that we anticipate. A hot cell was available, but it had to be cleared of radioactive sources and prepared for the operation of the IEC source. In addition, the main shield door was not operating and had to be repaired. (This repair was not funded from this project but from internal, infrastructure development funds.) Finally, we installed a 3 phase. 100 kilowatt, 280 volt line to provide the required power for the main accelerating power supply. (The remaining supplies and control systems operate from ordinary 115 volt, single phase service.)

Figure 6 is a picture of the outside of the hot cell. The 3 phase power breaker box is visible to the central right of the picture. The picture is looking through one of the main viewports of the hot cell, which is an oil-filled window 4 feet thick. Remote manipulators are also available. The electronics and control systems will be placed just outside the hot cell for convenient and safe operator access. They will connect to the IEC vacuum chamber with cables that snake through the access ports in the hot cell, where the IEC chamber itself is 
placed. We have already tested neutron leakage through the access ports using sensitive neutron detectors and isotopic sources. No detectable radiation was found. The IEC will be easily visible through the viewport.

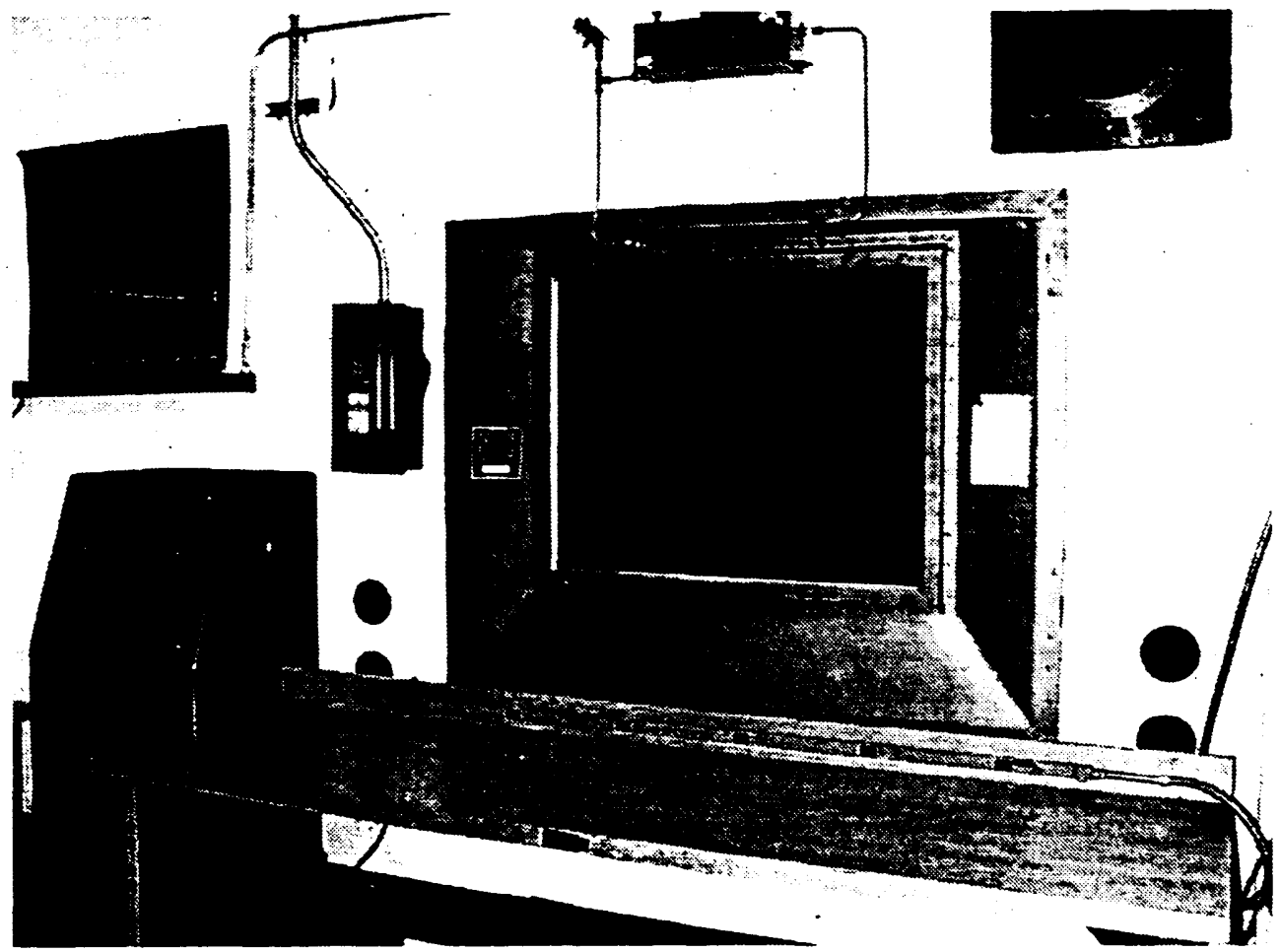

Figure 6: Picture of the outside of the shield cell at the location of the IEC experiment.

Negotiation and execution of the first CRADA agreement is in progress. The. CRADA will provide for a nonexclusive license of the basic IEC technology. However, the commercial. partner will develop an industrial version of the IEC, and this proprietary information will be protected. In return, the commercial partner will develop the industrial version of the IEC and will also develop the gas-handling for the Los Alamos IEC experiment. In this way, the Los Alamos research costs are mitigated.

The gas handling equipment is scheduled for the second year of operation and is specific to tritium operation. In the initial experiments the IEC will operate using pure deuterium, which can be vented in a normal vacuum system. This gas handling system is already in place and will be used for the experimental phases of IEC operation and optimization. However, once the IEC operating parameters are established, we will begin tritium operation. Tritium is a radioactive gas so that the system will be completely sealed and the vacuum system will no longer vent. Vacuum control and gas handling in a sealed system are very different problems than the pumped system that we now have in place. Vacuum and gas supply are maintained using metal "getters" that are heated. Our industrial partner, MF Physics, Inc., is a present supplier of conventional neutron generators and is an expert in sealed vacuum systems and getter operation. 


\section{Projections:}

Table 3 lists all of the original proposed milestones for the first year and their present status. All milestones have been met either on time or ahead of schedule. Ahead of schedule items are indicated by the bold and italicized for milestones 3 and 4.

Table 3: Project Schedule

\begin{tabular}{|c|c|c|c|}
\hline Milestone & $\begin{array}{l}\text { Date } \\
\text { Projected }\end{array}$ & $\begin{array}{l}\text { Date } \\
\text { Completed }\end{array}$ & Action \\
\hline 1 & $4 \operatorname{mos}$ & $4 \operatorname{mos}$ & $\begin{array}{l}\text { Procure all power supplies and vacuum } \\
\text { equipment. }\end{array}$ \\
\hline 2 & $8 \operatorname{mos}$ & $\begin{array}{l}\text { Not completed } \\
\text { yet }\end{array}$ & Execute CRADA agreement \\
\hline 3 & $8 \mathrm{mos}$ & $7 \mathrm{mos}$ & Complete construction of electrical systems. \\
\hline 4 & $10 \mathrm{mos}$ & $8 m o s$ & Build vacuum chamber \\
\hline 5 & $11 \mathrm{mos}$ & $\begin{array}{l}\text { Not completed } \\
\text { yet }\end{array}$ & Initiate experimental program \\
\hline
\end{tabular}

The execution of the CRADA agreement is due this month. It may be delayed by 1 to 2 months from the original projection. However, it will be completed within the 1997 scope.

All projected milestones and work will be completed within the 1997 scope. Some of the efforts are significantly ahead of schedule, such as the electrical and mechanical systems. We fully expect full operation 1 to 2 months ahead of schedule. The electrical system was completed 1 month ahead of schedule and the mechanical system will be completed 2 months ahead of schedule. Installation and initial operation will begin 2 months ahead of schedule.

\section{Funding:}

The funding levels have been within the projected levels. We will complete the 1997 scope fully within the original funding levels. The project will be completed within budget.

The present funding level from the last spreadsheet from our fiscal officer is:

\begin{tabular}{ll}
\hline $\begin{array}{l}\text { Funds spent and committed year to } \\
\text { date: }\end{array}$ & $\$ 275 \mathrm{k}$ \\
Total funds allocated: & $\$ 385 \mathrm{k}$ \\
Total funds remaining: & $\$ 110 \mathrm{k}$ \\
\hline
\end{tabular}

There are adequate funds to complete the assembly and installation of the equipment, and to begin initial operation.

\section{Issues/Problems:}

There have been two minor problems that we have encountered with this project. Both involve the financial accounting systems.

1. The first problem is very minor. The budget and accounting system has always lagged behind the true spending levels for this project. Although we use the laboratory system to accurately track costs, these costs are not fully reflected in the PTS summaries that 
are sent in by the program office. As a consequence, the program office has consistently under-reported the actual spending and this has disagreed with my reports. However, this small problem was easily solved by this explanation, and now the DOE and oversight organization know to trust the PTS reports accurate spending.

2. The second problem was also with funding. The carry over funding for this project was difficult to obtain and when it did arrive it was in the incorrect "TTP" account. As a consequence it was not immediately available. When it did become available, it was only available in a different account code. These difficulties caused some inconvenience and slight delays. Since then, the funds have been properly allocated and the work has resumed.

\section{Corrective Actions:}

The problems discussed above have been resolved as discussed above. Neither will have any averse effect on completion of this project in the first year. The project scope will be completed, on time, on budget, in the first year. 